\title{
EL CONOCIMIENTO INCONSCIENTE DEL TEMA Y EL DESEO DEL ANALISTA EN LA CLÍNICA DE DROGAS
}

\section{ARTÍCULO ORIGINAL}

ROCHA, Lorena Fabiani da ${ }^{1}$

ROCHA, Lorena Fabiani da. El conocimiento inconsciente del tema y el deseo del analista en la clínica de drogas. Revista Científica Multidisciplinar Núcleo do Conhecimento. Año 05, Ed. 04, Vol. 01, págs. 152-161. Abril de 2020. ISSN: 24480959, Enlace de acceso: https://www.nucleodoconhecimento.com.br/psicologiaes/conocimiento-inconsciente

\section{RESUMEN}

La atención clínica a los pacientes con drogas requiere que se observen las dimensiones inconscientes del sujeto que se droga y el analista acompañante, teniendo en cuenta que estas dimensiones preceden e implican la condición de dependencia del paciente. Por lo tanto, este trabajo tiene como objetivo aclarar las perspectivas psicoanalíticas que guían el enfoque del toxicomanfenómeno en el entorno de la atención. Conceptos como "inconsciente" y "deseo de analista" se tomaron con el fin de esclarecer cómo la situación analítica constituye un tratamiento individualizador y se opone al método clásico de la psiquiatría. Por lo tanto, se enfatiza la existencia de un conocimiento particular en cada paciente sobre su adicción a las drogas, que no fue predicho por la nosología médica. Por lo tanto, la técnica psiquiátrica se considera insuficiente y se explora un enfoque que busca interpretar mejor la adicción a las drogas, teniendo como herramienta para basar el deseo del analista. Además de los conceptos centrales aportados por el título, la obra también pasa por las nociones de "lenguaje", "malaise en la cultura", "yo", "monomanía", "castración" y "maduración de impulso". La investigación comenzó a partir del método

\footnotetext{
${ }^{1}$ Graduado en Psicología.
} 
de investigación bibliográfica, refiriéndose al tema "Adicción a las drogas" en el ámbito del psicoanálisis y la psiquiatría clásica. Se utilizó la literatura de autores consagrados que se confían en tales conocimientos, como Freud, Lacan y Bercherie.

Palabras clave: Adicción a las drogas, análisis, atención clínica, psiquiatría clásica.

\section{INTRODUCCIÓN}

La adicción a las drogas es un fenómeno que debe estudiarse más allá de la práctica de las drogas. En vista de esto, el psicoanálisis presupone una ética y opera a partir de un conocimiento inconsciente que produce alegría. El descubrimiento de este conocimiento nace de la experiencia de un proceso de análisis. Así, al admitir el deseo que contiene la última y particular verdad del sujeto, el método postulado por Sigmund Freud se opone a la forma en que la psiquiatría clásica de Pinel trató este fenómeno hasta principios de siglo. XX.

La presente investigación se guía por la teoría psicoanalítica, es decir, considera el descubrimiento del conocimiento inconsciente que afecta al ser que habla de una manera individual, lo que hace que la historia de la vida de cada sujeto sea una forma específica de habitar el lenguaje, en línea con su economía libidinal. Este lenguaje, a su vez, nos hace habitar el malestar de la cultura y nos enfrenta a objetos ofrecidos por el discurso del capitalista para amortiguar la falta irreparable que marca la existencia humana. En este sentido, el deseo del analista desafía el discurso de la ciencia que se ha incluido el tema del habla, el lenguaje, la subjetividad. Basándose en esta premisa, la investigación tiene como objetivo responder a la siguiente pregunta: ¿qué es velado por la adicción a las drogas, y cómo funciona el deseo del analista en la clínica de drogas?

Por lo tanto, el objetivo de este trabajo es señalar cómo el deseo del analista constituye una herramienta clínica y cómo se acerca a la intoxicación, desde el inconsciente y el impulso. La importancia de esta investigación es tomar el fenómeno en su doble valor social y subjetivo, expandiendo la visión reduccionista y el enfoque fenomenal del tema. Para cumplir el objetivo, el método utilizado en nuestro estudio 
fue la investigación bibliográfica de autores de renombre como Freud, Lacan y Bercherie, que abordan la drogadicción en el contexto del psicoanálisis y la psiquiatría clásica. La investigación fue el resultado del Proyecto de Iniciación Científica de la Universidad Veiga de Almeida (PIC UVA 2019) y guiado por la profesora y psicoanalista Clara Lúcia Inem.

\section{RESULTADOS}

Del presente estudio se dedujo el hallazgo de que todo el conocimiento proviene de la manipulación de un lenguaje que es pre-él (LACAN, 1954). Se trata del lenguaje humano, esencialmente simbólico y particular, por lo que aquí tiene posesión exclusiva de significado en el fenómeno toxicomaníaco. Así, esta investigación bibliográfica reafirma un método que se opone al modelo nosológico de la psiquiatría, sometifiando el fenómeno en cuestión.

Se notó que el lenguaje tiene primacía en el funcionamiento del sujeto y denuncia la intoxicación como un proceso secundario. A partir de ahí se entiende que la práctica de la droga vela el verdadero síntoma con las interpretaciones personales del paciente desde el inicio de su constitución. Se deduce, entonces, que el imperativo del tratamiento es la atribución de un simbolismo a las manifestaciones del sujeto (LACAN, 1955), y lo logramos, el simbolismo presente en la toxicomanización dice de una desilusión del paciente en relación con el otro (FREUD, 1927). Dicho esto, la condición de sujeción del paciente se aclara primero, y luego considera la práctica de drogas como un rompecabezas.

Por último, se observó el papel sustancial del deseo del analista en la gestión de este fenómeno. Esto se debe a que este deseo desiste, porque se ofrece como un lugar (QUINET, 2000) para instalar el deseo maduro del paciente. Es en esta dirección que el deseo del analista señala: en lugar de la repetición monótona y el impulso de cortocircuito promovido por la intoxicación, conducen al sujeto a un deseo maduro, que reconoce e invierte en sí mismo. Estas son las preguntas que se nos presentan: 
una intoxicación del lenguaje donde el significante falla y no permite que el cuerpo sea metafonizado por el conocimiento inconsciente.

\section{Discusíon}

\section{DE TOXICOMANIA COMO PRODUCTO DE UNA MALAISE SUBJETIVA EN LUGAR DE FIRMA EPISTEMOLOGICA}

A partir de una discusión sobre el posible origen de la religiosidad en "El malestar en la civilización", Freud (1930) especifica algunos mecanismos inconscientes que el ser humano engendra para lidiar con la angustia de habitar un mundo con el que descubre que no tiene relación de unidad, y que constituye un obstáculo en su búsqueda de la felicidad. Entre estos mecanismos participa la intoxicación, como un intento de recuperar una satisfacción vivida al comienzo de la vida, y que no poder ser recuperado, conduce a la repetición.

Por lo tanto, reconocemos que el yo actual del sujeto es lo que se desarrolló de su versión infantil (FREUD, 1930) después de una decepción. Sin embargo, el yo cubre una capa inconsciente, que en lugar de aparecer el sujeto como uno, dice de sus impulsos más pasivamente poner a prueba de la disección, como protegen el deseo. El fenómeno toxicomaníaco se toma entonces en relación con estos impulsos. Por lo tanto, es ineficaz observar y clasificar la práctica de drogas objetivamente como propone la psiquiatría clásica. Este método llevaría al analista a disfrutar del análisis cuando trae la droga como una marca de identidad. Así, como el analista no responde desde el lugar esperado por el paciente -es decir, el lugar de la enseñanza- haciendo que su acto sea tan desconocido como lo que se esconde bajo e/ propio sujeto, hay una doctrina analítica que alberga una brecha indispensable, que se opone al conocimiento de la psiquiatría, que ya existe agotada ante la experiencia con el paciente.

Para encontrar un camino alternativo, se entiende que el paciente primero debe confiar en la creencia de que el profesional sabe acerca de lo que trae como trastorno. 
Sin embargo, es sólo cuando el analista hace uso de tal suposición para hacer que el sujeto reconozca la causa en sí mismo, que uno encuentra el éxito indudable de un procedimiento.

Aquí se entiende que la intoxicación no es el síntoma, sino que vela con mecanismos simbólicos que ya tienen valor de palabra y llevan su propio significado (LACAN, 1953). Dicho esto, tomar otros caminos para descifrar el rompecabezas sólo avergonzaría a las cosas. Nombrar lo que ya lleva en sí mismo un significante, nos pone un nuevo camino fuera del sujeto, y por lo tanto sin salida.

Todavía - en el remora us Bercherie (1989), Pinel no prescindió de la formulación de una vasta nosografía que le hizo calificar la adicción a las drogas como locura de impulso, manía sin delirio, y finalmente, monomanía. Sin embargo, el psicoanálisis no consiente la actitud de poner fin a la comprensión de las adicciones a las drogas como impulsos de la causa moral, tomándolas únicamente por pasiones excesivamente prohibidas y duraderas. Aunque el toxicomanact se considera un intento de subvertir la cultura de la moderación de los impulsos, no se procede a dominarlos, por el contrario, se utiliza para que ascienden a mecanismos más maduros. Por lo tanto, el análisis investiga los afectos depositados en la droga, y la constancia en sí misma en la adicción a las drogas se toma como un efecto de la causa, nunca como la causa en sí.

La psiquiatría, a su vez, percibió las causas morales como las más numerosas y produciendo un tipo de "perturbación visceral" (BERCHERIE, 1989), propagando la locura del impulso por irradiación en el cuerpo. Es interesante notar que para el psicoanálisis, la causa del toxicomanfenómeno también hace un camino por irradiación, pero esta es la significativa irradiación en cadena que el núcleo discursivo del sujeto viajó hasta que fue observado clínicamente como un fenómeno.

En vista de esto, se encuentra en las consideraciones de Freud (1930), que para dilucidar la calidad de lo que actúa el deseo del analista, la práctica de la droga es aceptada en lo que tiene como objetivo proteger contra la frustración con el mundo 
real. La eficacia de los tóxicos radica en desensibilizar al paciente ante las desgracias de la vida neurótica, de modo que el fármaco subverta la química del cuerpo y hace que el sujeto disfrute incluso inmerso en el mundo hostil de la castración. Por lo tanto, el psicoanalista debe prestar atención al simbolismo latente en la eficacia de la droga, teniendo en cuenta que en la lucha por la felicidad, la intoxicación produce placer inmediato y una sensación de independencia del mundo externo, que se establece para promover el descontento.

En vista de esto, se considera que el propósito de la vida humana (FREUD, 1930) es precisamente la búsqueda de la felicidad y la búsqueda del placer. El sujeto pretende el éxito de la economía libidinal y trabaja para evitar el sufrimiento con el objetivo de la plena satisfacción. En este sentido, el principio del placer dicta rutas que apuntan a la homeostasis psíquica, incluso en sus apariencias más exageradas como la intoxicación. Así, este fenómeno constituye un intento de neutralizar la neurosis del sujeto, que está en este mundo sin poder deshacerse de él.

Los narcóticos actúan junto a impulsos que no se han sometido a la civilización y han tomado objetos prohibidos para satisfacerse. Teniendo en cuenta esta asociación, la situación analítica percibe una prohibición de los impulsos que podrían ser reprimidos y admite que este interdicto está más allá de la ley legal, a nivel subjetivo e inconsciente. Lo que se escanea es la historia edípica y particular de cada sujeto, en el que observan la interdicción simbólica y a veces lleva al sujeto a rebelarse contra la castración de impulsos. (FREUD, 1930).

En este trabajo se acuerda que, como principal fuente de sufrimiento para el sujeto, las relaciones humanas tienen repercusiones en la forma en que trata sus impulsos. A pesar de que el neurótico eligió vivir a través del amor (FREUD, 1927), las vicisitudes de las relaciones humanas pueden conducir al sujeto a opciones objetivas que lo prescinden del peligro del amor. La predilección por las figuras de la droga como una de estas opciones. Por lo tanto, se ocupa aquí del fenómeno toxicomaníaco como un subtipo de malestar que, a pesar de llegar comúnmente a los sujetos neuróticos, es sometido por cada uno a su manera. 
Dicho esto, el psicoanálisis considera que la costumbre nominalista de observar la práctica del narcotráfico en realidad sigue siendo insuficiente para explicar las consecuencias de la interdicción real. Es en este sentido que la técnica analítica transpone su enfoque a un terreno que no es observable a primera vista y no garantiza citas anticipadas, ya que no se trata de establecerse como un conocimiento absoluto. Por lo tanto, no es objetivo con el presente trabajo, dibujar un inventario completo de cómo los conceptos psicoanalíticos ofrecen solución al tema toxicomaníaco, sino por el contrario, rescatar la noción central de sujeto del inconsciente (FREUD, 1900) y cómo el analista lo convoca a través del fenómeno toxicomaníaco.

\section{EL CONOCIMIENTO INCONSCIENTE DEL TEMA}

Encontrar el instrumento con el que se aborda el tema de este trabajo, "La interpretación de los sueños" (FREUD, 1900, p. 406) trae nociones de que faltaba la psiquiatría de pinel. Aquí, se funda un tercer elemento sin el cual no proponemos considerar la adicción a las drogas. Entonces tomamos la palabra en lo que establece en sí mismo un lenguaje inconsciente y en sí mismo. Este elemento se puede representar mediante actos de repetición con valor simbólico de voz. En vista de esto, la reincidencia en las drogas se produce por la creencia del sujeto en la fiabilidad con la que el objeto lo hace revivir una satisfacción pasada, y apunta a un lenguaje inconsciente que subyace a esta satisfacción.

Para desafiar el placer en esta repetición, la relación con el objeto se considera como una relación irrevocable de falta de objeto (LACAN, 1956). Esta relación entre el paciente y el objeto no es directa, sino transpuesta a través de un agujero. Debido a esto, no se puede tomar como el centro de la situación analítica, pero se utiliza para la falta de ser revelado al sujeto.

Dicho esto, el analista interpone sus interpretaciones no en ningún momento, pero donde la derivada de la falta de significante - la droga - simula amortiguar el agujero. Por lo tanto, uno no apunta directamente a la intoxicación, sino al proceso simbólico que cubre. El concepto de castración (LACAN, 1956) se aborda entonces como un 
desarrollo de la insuficiencia mostrada por el significante del Otro en la constitución del sujeto. Por lo tanto, se entiende que el poder del tratamiento está en la conciencia oportunista de que, si incluso el Otro en relación con quien el paciente se sometió a sí mismo, lo llevó al desencanto, el sustituto que trata de cumplir su función tampoco tuvo éxito.

Por lo tanto, tomando el inconsciente como causa, el analista lleva al paciente fijado en la droga para concluir que incluso los tóxicos cuando hacen que el cuerpo disfrute apareciendo para silenciar la castración, no son capaces de aniquilar los efectos de la misma.

Además, a pesar de considerar las relaciones de objetos como fundamentalmente perforadas, sin poder enfocarse en ellas, es desde el agujero que estructura la búsqueda en la relación con la droga, ese deseo se encuentra como una verdad donde el analista se levanta eficazmente. Además, debe entenderse que primero el deseo afecta al sujeto y al sujeto ya afectado por una causa, afecta y elige el objeto (LACAN, 1958). En esta relación, al igual que el objeto, el deseo en sí tampoco tiene un representante real, sin embargo, ya que se encuentra en el propio sujeto y no fuera, tiene posibilidades de salidas más auténticas para dar cuenta de la castración. Entonces se basa en la relación de hiance con el objeto de la droga con la promoción del deseo, sin ignorar que incluso lo que es más auténtico en el tema no se alcanza sin obstáculos.

Por lo tanto, como propuesto por Lacan (1959), este estudio toma la fantasía fundamental del sujeto como la forma fiel de la relación objeto. El valor de esta fantasía en el enfoque del fenómeno discutido aquí es demostrar no sólo el sujeto neutralizado y la droga de la que utiliza como objeto de obstrucción, sino sobre todo el deseo que mueve su aparato psíquico. Al ser capaz de situarse en su propia fantasía a través de su deseo, el sujeto puede emerger como verdad, en lugar de un eco del deseo de otra persona que una vez lo cautivó por el lenguaje. Así, el paciente reconocerá un deseo fuera de sí mismo, y que el suyo no admita objeto de satisfacción, entrando así en otra etapa de su constitución como ser: la maduración del impulso. 
Por otra parte, la intoxicación como una inversión defectuosa para nombrar la situación del sujeto en el mundo simbólico, abre un espacio para el surgimiento del deseo, ante el cual este sujeto volverá a intentar una cita que también fracasará (QUINET, 2000). El análisis proporcionará al sujeto el discurso analítico que trata de esta inviabilidad. Es decir, la subversión del verdadero síntoma se encuentra en todos los casos, en el propio sujeto, ya que mantiene con el real de sí mismo la oposición a lo que lo constituyó simbólica e imaginativamente.

Dicho esto, el analista recomienda no erradicar la práctica de las drogas, a fin de localizar dónde la satisfacción del sujeto con el objeto primero se aloja. La elección de la droga parece garantizar que el paciente ha encontrado una manera de disfrutar de que independientemente de la que un día vino frustración - el Otro. Sin embargo, lo que Santiago (2001) propone es que en el acto toxicomaníaco el sujeto no parece buscar un objeto sustituto para su relación con el Otro. Por lo tanto, el autor argumenta que el llamado "adicto a las drogas" implica tomar la alegría fálica como solución en sí misma, cuando demuestra que ha encontrado un objeto no genital que satisface su búsqueda y descalifica la castración.

En resumen, tomando la palabra del sujeto por ferrocarril, se descubre que el principio rector de la práctica de las drogas no sólo muestra la maximización del placer y la eliminación del disgusto como un objetivo práctico cerrado en sí mismo. Hay mientras tanto la dinámica de la unidad con el objeto, una alegría que se justifica en la historia - y sólo allí - del sujeto. Por lo tanto[...], el "sujeto como habla puede encontrar enteramente su respuesta, su regreso, su secreto, su misterio, en el símbolo construido". (LACAN, 1954, p. 252).

\section{EL DESEO DEL ANALISTA}

Aquí está el último operador del análisis que trataremos: el deseo del analista. Después de que la investigación haya indicado lo primordial que es centrarse en el conocimiento inconsciente del sujeto y su deseo de la misma calidad, mostrará las peculiaridades e implicaciones de este otro deseo. En este sentido, a pesar del deseo 
del analista de presentarse a lo largo del análisis, se considera que es después de oporturinizar la apariencia del conocimiento inconsciente que abre el camino para que el sujeto se lance en otra etapa. Así, después de que el paciente ha tomado el tratamiento para lo que dirige su satisfacción en el fármaco a su pulsación, el analista con su deseo atento (LACAN, 1960), también provoca el reconocimiento por parte del sujeto sobre las características de su propio deseo.

Dicho esto, después del momento en que el analizador logró sostener el diálogo analítico y la imposibilidad de nombrar su condición deseable, se le llevará a percibir este deseo como lo que está vinculado por la ley, como el deseo del Otro. El deseo del analista trabaja para impulsar al sujeto a la transgresión de la ley del Otro en lo que no significa el incumplimiento del derecho constitucional de la sociedad. De hecho, promueve en el análisis, la des-subjeción a un estatuto simbólico que terminó desregulando el funcionamiento auténtico de este tema. Así, ante las verdaderas causas de la práctica de la droga, el paciente se enfrenta a la incoherencia del Otro de la Ley (QUINET, 2000), sin embargo, no se deja a la suerte, ya que el deseo del analista comienza a ocupar el lugar de impotencia, pero sin el Otro, sin la Ley.

Por lo tanto, incluso si este deseo toma el lugar de la incoherencia, él mismo mantiene un acertijo (QUINET, 2000). Si el sujeto busca lo que el analista piensa sobre su adicción a las drogas o lo que su conocimiento propone para este fenómeno, no encontrará juicio, haciendo imposible interpretar este deseo. Esto se debe a que el análisis funciona de manera que cada uno juzga por sí mismo, sólo haciendo evidente el carácter de estas elecciones para que el sujeto no decida sobre su propia ignorancia. Por lo tanto, es el conocimiento del tema lo que encuentra el deseo del analista, y no al revés, como sugiere la psiquiatría clásica.

Además, la técnica analítica ni siquiera tiene como objetivo "tratar la adicción a las drogas", ya que el deseo del analista está en otra parte, aparte del de curación, liberación, purga o ayuda a los demás. Se opone, por ejemplo, al trato moral propuesto por Pinel para dar cuenta de la reorientación de los equivocados a la razón (BERCHERIE, 1989). Si bien la psiquiatría se ha instrumentalizado en las instituciones 
represivas y la idea de que la mente del paciente se vio afectada por sus percepciones, el analista también considera el medio ambiente y la realidad psíquica del sujeto, pero apuesta por un espacio de habla y la (re)constitución simbólica del inconsciente.

Finalmente, el deseo del analista admite el deseo inconsciente del sujeto, pero difiere de él en que no responde a ninguna demanda, y ofrece espacio para el deseo del sujeto también de no preparar respondiendo. El deseo del analista no habla de un deseo personal de saber, sino que es el lugar de un know-the-less (LACAN, 1969), una posición que existe únicamente para la causa. Así, este deseo habla del objetivo supremo de permitir la existencia del ser, separándolo de los lazos inconscientes contraídos por herencia simbólica.

\section{CONCLUSIONES}

Este trabajo ha llevado a cabo una considerable investigación teórica que concluye, sin embargo, con una idea concisa: que el fenómeno tiene una historia y esta es la historia personal del sujeto que se droga. Por lo tanto, el deseo del analista es un motor, no de cura, sino de cuestionar la repetición con precedentes en el propio repetidor. Es como en toda experiencia de análisis, una clínica de descubrimiento, pero no de conocer a priori o deseo de sanar, en la que el paciente construye sus propios caminos (LACAN, 1960) para el resultado de la repetición. En vista de esto, la relevancia de este trabajo se extrae en lo que reitera una técnica que valora la autonomía del sujeto en la práctica de drogas.

La investigación nos lleva a inducir que, como cualquiera de los pacientes, lo que busca al sujeto que se droga a sí mismo es la felicidad. Teniendo esto en cuenta, ¿cuál es la situación analítica que se plantea es "Cruzada por qué parámetros busca el sujeto? ¿Qué sabe él acerca de la naturaleza de esta felicidad deseada, por qué hace lo que hace para lograrla?"

Así, tomamos la relevancia del fenómeno toxicomaníaco en el que su estudio contribuye socialmente y a la clínica uno por uno, sin entregarse a las generalizaciones, incluso ante la recurrencia del fenómeno. El deseo del analista 
funciona para arrojar luz sobre un conocimiento inconsciente y particular. En este sentido, en la medida en que el yo es un caso de ignorancia en el sujeto porque se refiere al Otro, una esfera más íntima está orientada a alcanzar su verdad. Para ello, se toma el siguiente camino: el discurso como acceso a la verdad inconsciente del sujeto frente a la intoxicación. Por lo tanto, se admite no saber a priori sobre lo que el discurso del sujeto se trata (LACAN, 1956), pero se toma como una ruta para el acceso a más allá del fenómeno toxicomaníaco.

\section{REFERENCIAS}

BERCHERIE, Paul. Os fundamentos da clínica: história e estrutura do saber psiquiátrico. Rio de Janeiro: Jorge Zahar, 1989.

FREUD, Sigmund. A interpretação dos sonhos, parte I. Edição Standard Brasileira das Obras Completas de Sigmund Freud, vol. IV. Rio de Janeiro: Imago,1996.

. A interpretação dos sonhos, parte II. Edição Standard Brasileira das Obras Completas de Sigmund Freud, vol. V. Rio de Janeiro: Imago,1996.

. O Mal Estar na Civilização. Edição Standard Brasileira das Obras Completas de Sigmund Freud, vol. XXI. 2. ed. Rio de Janeiro: Imago, 1988. LACAN, Jacques. O seminário, livro 1: os escritos técnicos de Freud. 2. ed. Rio de Janeiro: Jorge Zahar, 2009.

O seminário, livro 2: o eu na teoria de Freud e na técnica da psicanálise. 2. ed. Rio de Janeiro: Jorge Zahar, 2010.

. O seminário, livro 4: a relação de objeto. Rio de Janeiro: Jorge Zahar, 1995.

. O seminário, livro 6: o desejo e sua interpretação. 1. ed. Rio de Janeiro: Jorge Zahar, 2016. 
. O seminário, livro 7: a ética da psicanálise. Rio de Janeiro: Jorge Zahar, 2008.

O seminário, livro 11: os quatro conceitos fundamentais da psicanálise. 2. ed. Rio de Janeiro: Jorge Zahar, 2008.

O seminário, livro 17: o avesso da psicanálise. Rio de Janeiro: Jorge Zahar, 1992.

QUINET, Antonio. A descoberta do inconsciente: do desejo ao sintoma. 7. ed. Rio de Janeiro: Jorge Zahar, 2018.

SANTIAGO, Jésus. A droga do toxicômano: uma parceria cínica na era da ciência. 2. ed. Belo Horizonte: Relicário Edições, 2017.

Enviado: Marzo de 2020.

Aprobado: Abril de 2020. 